\title{
Strong green luminescence in quaternary InAIGaN thin films
}

\author{
Shih-Wei Feng, Yung-Chen Cheng, Yi-Yin Chung, and C. C. Yang ${ }^{\text {a) }}$ \\ Graduate Institute of Electro-Optical Engineering and Department of Electrical Engineering, \\ National Taiwan University, 1, Roosevelt Road, Sec. 4, Taipei, Taiwan, Republic of China \\ Kung-Jeng $\mathrm{Ma}$ \\ Department of Mechanical Engineering, Chung Hua University, Hsinchu, Taiwan, Republic of China \\ Chih-Chiang Yan and Chen Hsu \\ Department of Mechanical Engineering, Chung Cheng Institute of Technology, National Defense University, \\ Tahsi, Taoyuan, Taiwan, Republic of China \\ J. Y. Lin and H. X. Jiang \\ Department of Physics, Kansas State University, Manhattan, Kansas 66506-2601
}

(Received 23 July 2002; accepted 10 January 2003)

\begin{abstract}
We observed strong green luminescence (around $2.37 \mathrm{eV}$ ) in two InAlGaN thin film samples, which were originally prepared for applications in the UV range. Based on the observation of the InN peaks and the extended distributions, corresponding to InGaN compounds, in the x-ray diffraction patterns, such green luminescence was attributed to the formation of indium-rich clusters, which formed localized states, in the quaternary films of low indium content (2\%). Carrier trapping of such localized states, particularly when carriers received thermal energy such that they could overcome certain potential barriers around the clusters, resulted in strong green luminescence. The observed optical behaviors, including the blueshift phenomenon of photoluminescence spectral peak position in varying temperature, showed that the green luminescence was quite different from the previously reported yellow luminescence, which resulted from defects. () 2003 American Institute of Physics.
\end{abstract}

[DOI: $10.1063 / 1.1556965]$

Recently, quaternary InAlGaN-based UV photodetectors showing high photoresponse have been demonstrated. ${ }^{1,2} \mathrm{Be}-$ sides the application to photodetectors, quaternary InAlGaN compounds were used in InGaN/InAlGaN quantum well (QW) light emitters ${ }^{3}$ and GaN/InAlGaN heterojunction fieldeffect transistors. ${ }^{4}$ With all these successful implementations of InAlGaN-based devices, the material properties as well as optical characteristics of such a compound have not been well studied yet. In particular, the visible luminescence behaviors in an InAlGaN compound with the band gap in the UV range have never been well discussed although photon emission of deep levels was briefly mentioned. ${ }^{5}$ These deep levels may originate from indium aggregation and phase separation, which were widely reported in $\mathrm{InGaN} / \mathrm{GaN} \mathrm{QW}$ structures. ${ }^{6,7}$

In this letter, we report the strong green luminescence in two quaternary InAlGaN samples, which were originally prepared for applications in the UV range, with the results of material and optical analyses. X-ray diffraction (XRD), temperature-dependent photoluminescence (PL), photoluminescence excitation (PLE), and high-excitation PL measurements were performed. Based on the measurement results, we propose the existence of InGaN clusters in InAlGaN quaternary alloys. Such clusters are responsible for the strong green luminescence.

The two InAlGaN samples used in this study were grown in a low-pressure metalorganic chemical vapor deposition reactor. In sample KSU-667, a $25 \mathrm{~nm}$ low-temperature

${ }^{a)}$ Electronic mail: ccy@cc.ee.ntu.edu.tw grown $\mathrm{GaN}$ buffer layer was first deposited on the sapphire substrate, followed by the deposition of $\sim 1 \mu \mathrm{m} \mathrm{GaN}$ layer. A quaternary InAlGaN film with a thickness of about $100 \mathrm{~nm}$ was then deposited on the top of the GaN layer. In sample KSU-669, an about $100 \mathrm{~nm}$ InAlGaN film was directly deposited on a sapphire substrate with a $25 \mathrm{~nm} \mathrm{GaN}$ buffer layer. The compositions of $\mathrm{In}$ and $\mathrm{Al}$ were estimated to be $2 \%$ and $15 \%$, respectively, in both samples. The details of growth procedures were reported previously. ${ }^{1}$ The standard PL and PLE measurements were carried out. For high excitation PL measurements, the fourth harmonic $(266 \mathrm{~nm})$ of a $Q$-switch Nd:yttritium-aluminum-garnet laser was used as the excitation source.

Figures 1(a) and 1(b) show the XRD patterns of samples KSU-667 and KSU-669, respectively. The diffraction peaks could be well identified. ${ }^{8-11}$ In sample KSU-667, the clear $\mathrm{GaN}$ peaks of different orders originate mainly from the 1 $\mu \mathrm{m}$ GaN layer. Such peaks are not clearly seen in sample KSU-669. The InN peaks (see the insets), corresponding to the indium-rich phase, provide a direct evidence of indium aggregation or phase separation. The broad distributions (indicated with arrows in the insets) between the InN (0002) peak and the $\mathrm{GaN}$ (0002) peak represent strong indium compositional fluctuations of broad variations in size, shape, and indium content in the two samples, particularly in KSU-667.

Figures 2(a) and 2(b) show the PL data with high-power excitation and PLE spectra of KSU-667 and KSU-669, respectively. The average excitation power increases from 50 to $150 \mathrm{~mW}$ for the five PL curves. Besides peaks A and B in the PL spectra, corresponding to InAlGaN and GaN, respectively, there is strong green luminescence (peak C) in both 


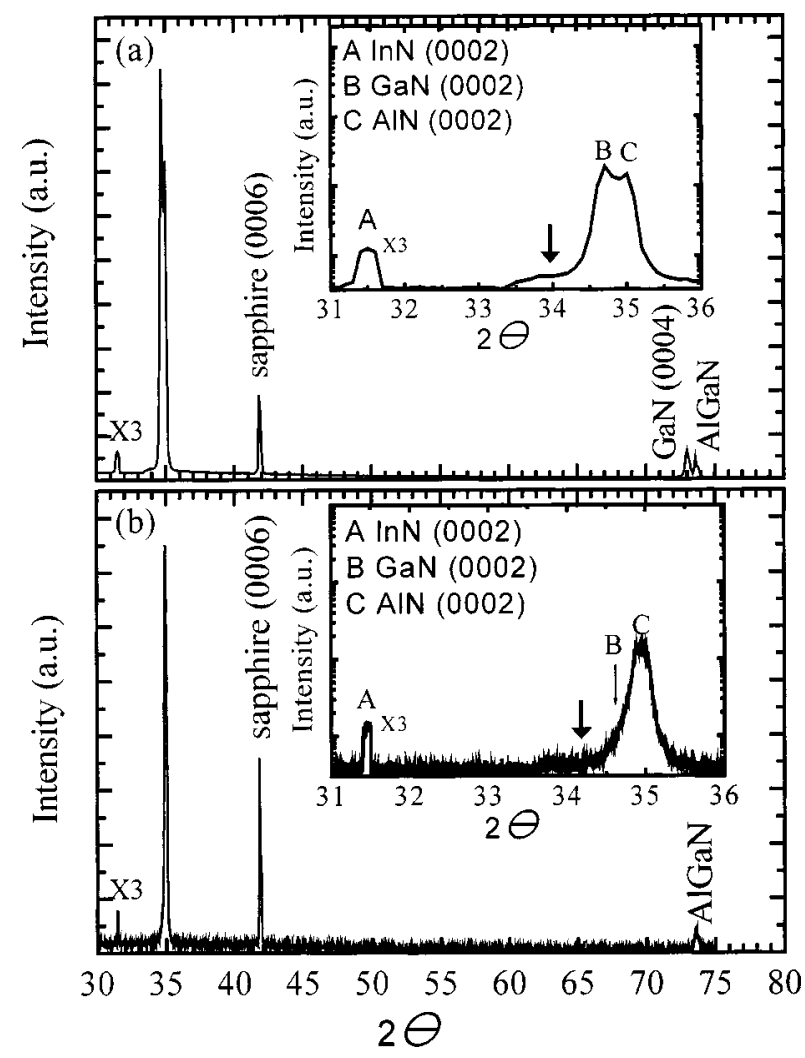

FIG. 1. XRD patterns of samples KSU-667 (a) and KSU-669 (b). Insets of (a) and (b) show fine-scale patterns.

samples. The broad spectral widths in the green range imply that the formed InGaN clusters may have large variations in their geometries and compositions. Comparing the relative intensity and spectral width of peak $\mathrm{C}$ with peak $\mathrm{A}$, one can see that the green emission of KSU-667 is relatively stronger and spectrally broader than that of KSU-669. This trend is consistent with the higher degree of indium aggregation in KSU-667, as shown in Fig. 1. The existence of the thick GaN layer in sample KSU-667 is responsible for the strong emission of the B band. To observe the Stokes shift (SS) of green luminescence, the PLE detection photon energy was set at 10 $\mathrm{meV}$ below the individual PL peak in each sample. The PLE spectrum of KSU-667 shows the absorption peaks of quaternary InAlGaN, GaN, and InGaN around 3.8, 3.5, and $2.6 \mathrm{eV}$, respectively. The SS of green luminescence in each sample can be observed. It stands for another evidence of geometry and composition variations in indium aggregation.

To further understand the behaviors of green luminescence, we used a $\mathrm{He}-\mathrm{Cd}$ laser as the excitation source for temperature-dependent PL measurements. Figures 3(a) and 3(b) show the temperature-dependent PL spectra of KSU-667 and KSU-669, respectively. One can see that the PL intensity increases with temperature up to $225 \mathrm{~K}$ in KSU-667. Meanwhile, that of KSU-669 is basically maintained constant. The temperature-dependent PL results are summarized in Figs. 4(a) and 4(b), in which the normalized integrated PL intensities and PL spectral peak positions of the two samples are shown. In KSU-667, the integrated PL intensity increases up to $225 \mathrm{~K}$ first and then decreases with increasing temperature. On the other hand, it is almost temperature independent in KSU-669. Such behaviors are quite different from previous results, which were usually observed in typical III-V

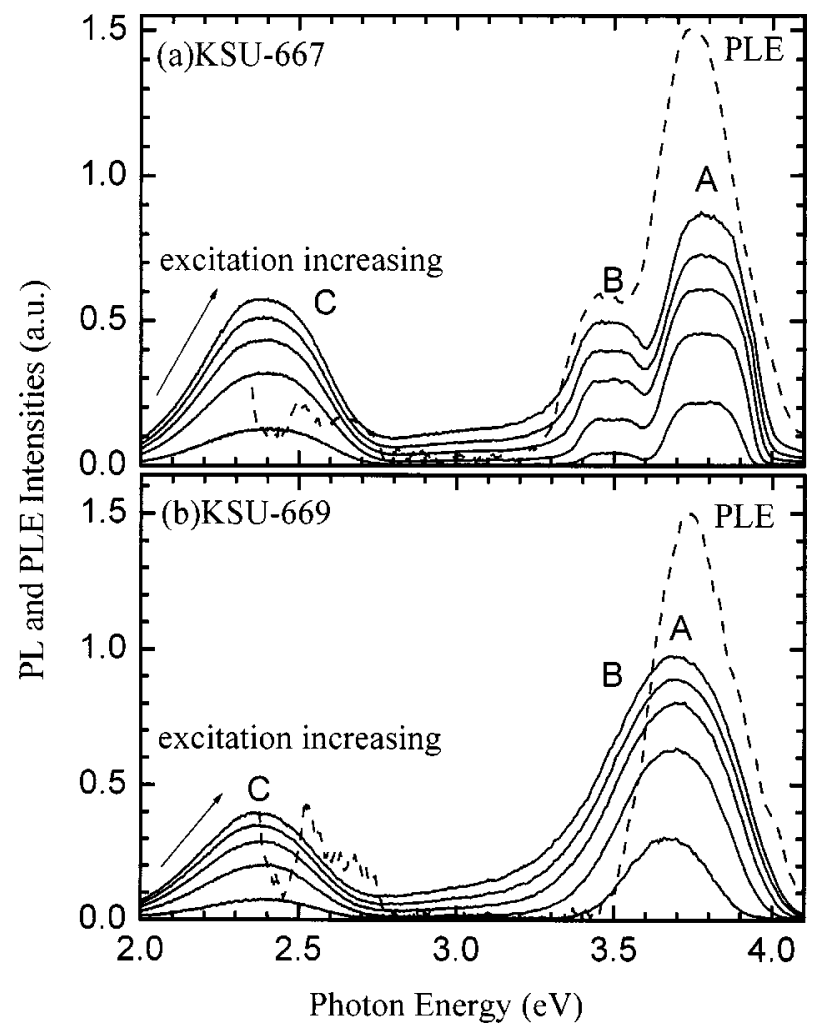

FIG. 2. High excitation PL (solid lines) and PLE (dashed lines) spectra of samples KSU-667 (a) and KSU-669 (b). The average excitation powers for the five solid curves are set at 50,75,100,125, and $150 \mathrm{~mW}$.

compounds. In such a compound, integrated PL intensity always decays with temperature due to the increasing nonradiative recombination. Such an anomalous phenomenon can be attributed to carrier supply with thermal energy. With thermal energy, carriers can overcome certain potential barriers and relax into localized states formed by the InGaN clusters. ${ }^{12}$ In other words, when carriers are generated in the states of $\mathrm{GaN}$ and InAlGaN, most of them would not relax to the potential minima of the localized states unless thermal energy is supplied. In KSU-667, because the prominent indium aggregations provide more localized states, the carrier capture process is stronger, leading to the increasing trend up to $225 \mathrm{~K}$. Beyond this temperature, nonradiative process dominates. The almost temperature-independent integrated PL intensity of KSU-669 suggests the equilibrium between carrier supply and loss. Carrier loss can be due to nonradiative recombination and carrier escape with thermal energy.

The temperature-dependent PL spectral peak positions in Fig. 4(b) show a double $S$-shape variation (redshiftblueshift—redshift—blueshift) in KSU-667 as temperature increases. Single $S$-shape behaviors were widely observed in InGaN/GaN QW structures. They were attributed to the temperature-dependent carrier dynamics associated with carrier localization in potential minima. ${ }^{13}$ The double $S$-shape variation may imply that there exist two groups of indiumrich clusters of different potential barriers for carrier trapping. The first blueshift $(40 \mathrm{~K}<T<120 \mathrm{~K})$ can be attributed to the band filling effect of the first group of localized states. The second blueshift $(200 \mathrm{~K}<T<250 \mathrm{~K})$ is due to the same effect of the second group of higher potential barriers. When the band filling is saturated, the phonon effect (redshift) 


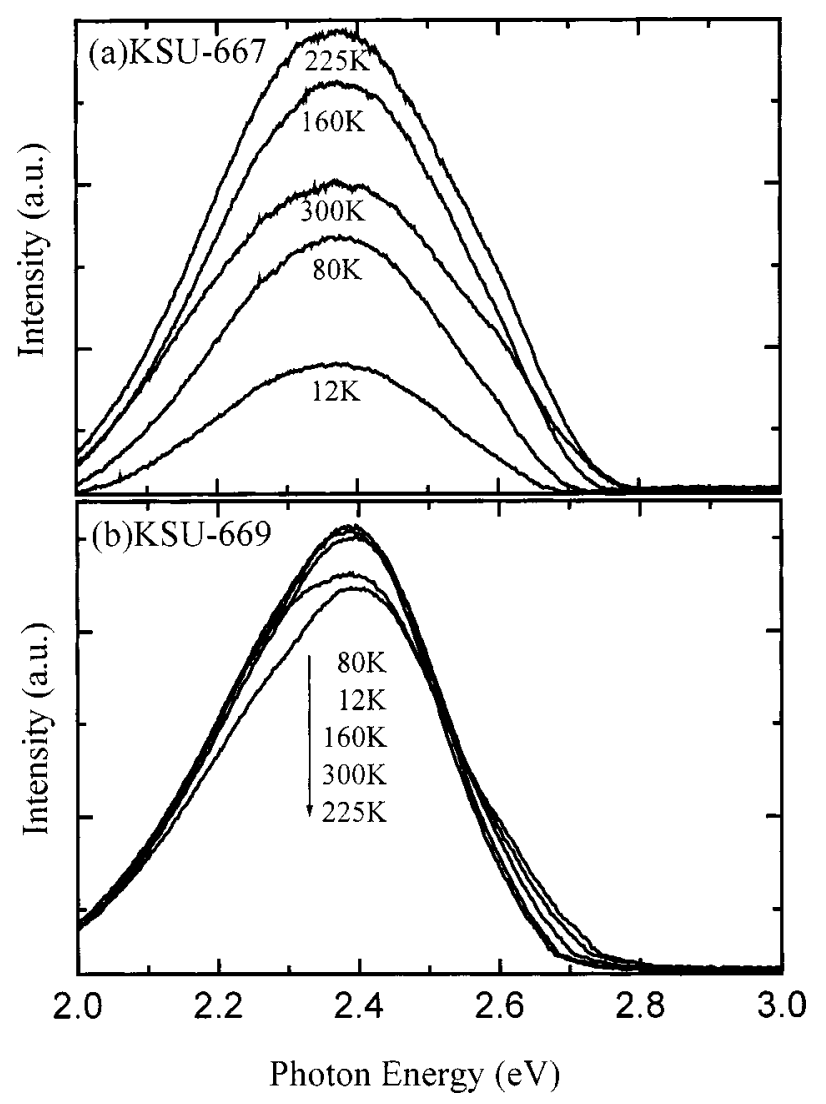

FIG. 3. PL spectra at several temperatures of samples KSU-667 (a) and KSU-669 (b).

dominates. As for sample KSU-669, the PL spectral peak positions exhibit blueshift first and then redshift as temperature increases. This one-step blueshift may imply that only one type of indium-rich clusters exists in this sample.

The properties of the observed strong green luminescence are quite different from those of the yellow luminescence previously reported. The following differences are worth noting: First, the emission peak of the green luminescence (at $12 \mathrm{~K}$ ) is around $2.37 \mathrm{eV}(523 \mathrm{~nm})$, while that of yellow luminescence is around $2.21 \mathrm{eV}(560 \mathrm{~nm}){ }^{14,15} \mathrm{Sec}-$ ond, the PL spectral peak positions of yellow luminescence did not exhibit the $S$-shape variation with temperature, particularly the blueshift behaviors. Based on the measured optical characteristics and XRD results, it is believed that the observed strong green luminescence originates from the formation of indium-rich clusters, instead of defects, in the InAlGaN thin films. ${ }^{15,16}$ The XRD patterns shown in Fig. 1 did not show the lattice structures corresponding to the band gaps of the observed green luminescence. It is believed that certain microstructures, consisting of local $\mathrm{InN}$ and $\mathrm{InGaN}$ lattices, in the samples (such as quantum dot-like clusters) are formed such that energy states, corresponding to green luminescence, exist.

In summary, we have observed strong green luminescence (around $2.37 \mathrm{eV}$ ) in two InAlGaN thin film samples, which were prepared for applications in the UV range. Such green luminescence was attributed to the formation of indium-rich clusters, which formed localized states, in the quaternary films.

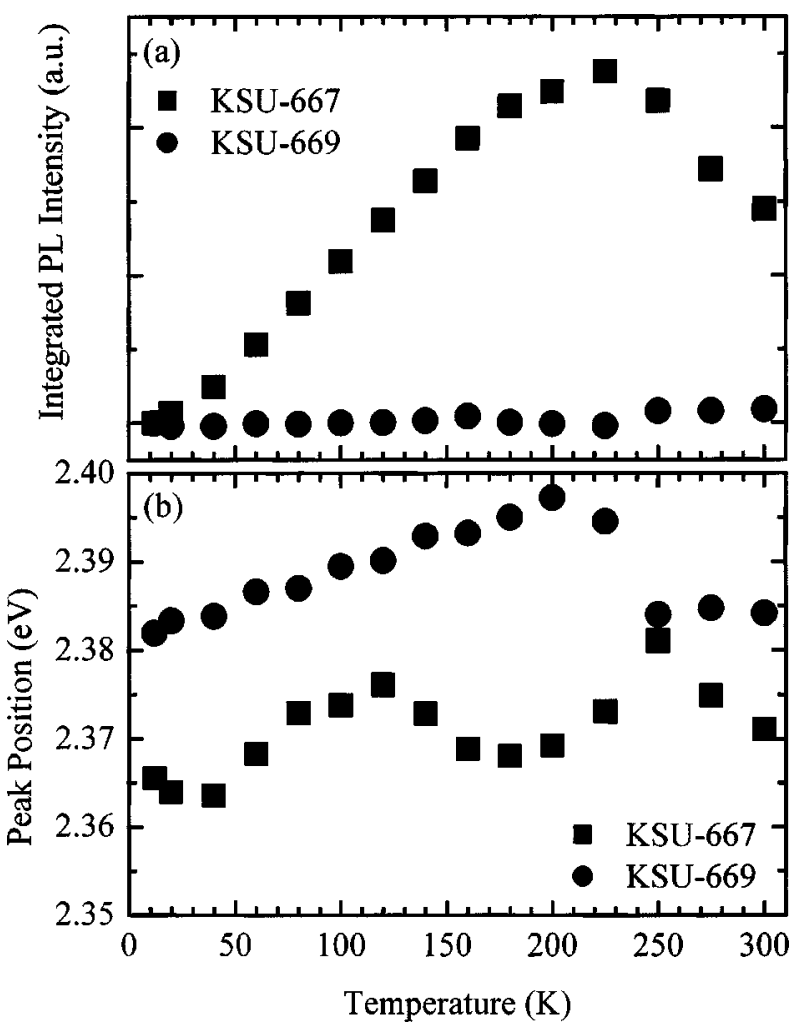

FIG. 4. (a) Integrated PL intensity and (b) PL spectral peak position as functions of temperature of samples KSU-667 and KSU-669.

This research was supported by National Science Council, The Republic of China, under Grants Nos. NSC 90-2112M-002-052, NSC 90-2215-E-002-027, and NSC 90-2215-E002-041.

${ }^{1}$ T. N. Oder, J. Li, J. Y. Lin, and H. X. Jiang, Appl. Phys. Lett. 77, 791 (2000).

${ }^{2}$ J. Li, K. B. Nam, K. H. Kim, J. Y. Lin, and H. X. Jiang, Appl. Phys. Lett. 78, 61 (2001).

${ }^{3}$ M. E. Aumer, S. F. LeBoeuf, S. M. Bedair, M. Smith, J. Y. Lin, and H. X. Jiang, Appl. Phys. Lett. 77, 821 (2000).

${ }^{4}$ M. Asif Khan, J. W. Yang, G. Simin, R. Gaska, M. S. Shur, H. C. Zur Loye, G. Tamulaitis, A. Zukauskas, D. J. Smith, D. Chandrasekhar, and R. Bicknell-Tassius, Appl. Phys. Lett. 76, 1161 (2000).

${ }^{5}$ F. G. McIntosh, K. S. Boutros, J. C. Roberts, S. M. Bedair, E. L. Piner, and N. A. El-Masty, Appl. Phys. Lett. 68, 40 (1996).

${ }^{6}$ Y. Narukawa, Y. Kawakami, M. Funato, S. Fujita, S. Fujita, and S. Nakamura, Appl. Phys. Lett. 70, 981 (1997).

${ }^{7}$ Y. S. Lin, K. J. Ma, C. Hsu, S. W. Feng, Y. C. Cheng, C. C. Liao, C. C. Yang, C. C. Chou, C. M. Lee, and J. I. Chyi, Appl. Phys. Lett. 77, 2988 (2000).

${ }^{8}$ W. J. Meng and T. A. Perry, J. Appl. Phys. 76, 7824 (1994).

${ }^{9}$ Q. X. Guo, T. Yamamura, A. Yoshida, and N. Itoh, J. Appl. Phys. 75, 4927 (1994).

${ }^{10}$ A. Saxler, P. Kung, C. J. Sun, E. Bigan, and M. Razeghi, Appl. Phys. Lett. 64, 339 (1994).

${ }^{11}$ M. D. McCluskey, L. T. Romano, B. S. Krusor, D. P. Bour, N. M. Johnson, and S. Brennan, Appl. Phys. Lett. 72, 1730 (1998).

${ }^{12}$ S. W. Feng, Y. C. Cheng, Y. Y. Chung, C. C. Yang, M. H. Mao, Y. S. Lin, K. J. Ma, and J. I. Chyi, Appl. Phys. Lett. 80, 4375 (2002).

${ }^{13}$ Y. H. Cho, G. H. Gainer, A. J. Fischer, J. J. Song, S. Keller, U. K. Mishra, and S. P. DenBarrs, Appl. Phys. Lett. 73, 1370 (1998).

${ }^{14}$ I. H. Lee, I. H. Choi, C. R. Lee, and S. K. Noh, Appl. Phys. Lett. 71, 1359 (1997).

${ }^{15}$ G. Li, S. J. Chua, S. J. Xu, W. Wang, P. Li, B. Beaumont, and P. Gibart, Appl. Phys. Lett. 74, 2821 (1999).

${ }^{16}$ E. F. Schubert, I. D. Goepfert, and J. M. Redwing, Appl. Phys. Lett. 71, 3224 (1997) 
Applied Physics Letters is copyrighted by the American Institute of Physics (AIP). Redistribution of journal material is subject to the AIP online journal license and/or AIP copyright. For more information, see http:/ojps.aip.org/aplo/aplcr.jsp

Copyright of Applied Physics Letters is the property of American Institute of Physics and its content may not be copied or emailed to multiple sites or posted to a listserv without the copyright holder's express written permission. However, users may print, download, or email articles for individual use. 